\title{
Immunogenicity and Safety of Combined Tetanus, Reduced Diphtheria, Acellular Pertussis Vaccine when Co-Administered with Quadrivalent Meningococcal Conjugate and Human Papillomavirus Vaccines in Healthy Adolescents
}

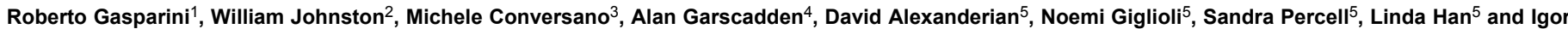 \\ Smolenov $5^{*}$ \\ ${ }^{1}$ Department of Health Sciences, University of Genoa, Genoa, Italy \\ ${ }^{2}$ Birmingham Pediatrics, Birmingham, AL, USA \\ ${ }^{3}$ Taranto Local Health Unit, Department of Prevention, Taranto, Italy \\ ${ }^{4}$ Clinical Research Advantage, Colorado Springs Health Partners, Colorado Springs, CO, USA \\ ${ }^{5}$ Novartis Vaccines and Diagnostics, Inc., Cambridge, MA, USA \\ "Corresponding author: Igor Smolenov, Novartis Vaccines, Hullenbergweg 83-85, P.O. Box 23023, NL-1100 DM, Amsterdam, Netherlands, Tel: +31 20 5640551; Fax: \\ +31 20 5640565; E-mail: igor.smolenov@novartis.com
}

Received date: 10 April 2014; Accepted date: 21 May 2014; Published date: 24 May 2014

Copyright: (C) 2014 Gasparini R, et al. This is an open-access article distributed under the terms of the Creative Commons Attribution License, which permits unrestricted use, distribution, and reproduction in any medium, provided the original author and source are credited.

\section{Abstract}

Objective: Current United States immunization recommendations for adolescents include vaccines against tetanus, diphtheria and pertussis (Tdap), human papillomavirus (HPV), and Neisseria meningitidis serogroups A, C, $\mathrm{W}-135$, and $\mathrm{Y}$. In this Phase IV study, we primarily investigated the impact of concomitant administration of a quadrivalent meningococcal $\mathrm{CRM}_{197}$-conjugate vaccine (MenACWY-CRM) with Tdap and HPV vaccines, in terms of immunogenicity to Tdap antigens and overall reactogenicity.

Methods: A total of 801 healthy adolescents aged 10-18 years were randomized to one of two groups to receive either MenACWY-CRM or a placebo, co-administered with Tdap and a quadrivalent HPV vaccine (HPV4). Antibody responses to the Tdap antigens, as well as to meningococcal serogroups $A, C, W-135$, and $Y$, were assessed at one month post-vaccination. Safety and adverse events were monitored throughout the study.

Results: One month post-vaccination, $95 \%$ and $99 \%$ of subjects in the MenACWY-CRM group had seroprotective antibody levels $(\geq 1.0 \mathrm{lU} / \mathrm{mL})$ against the diphtheria and tetanus toxoids, respectively, compared with $82 \%$ and $98 \%$ in the placebo group. Ratios of geometric mean concentrations of antibodies against pertussis antigens pertussis toxin, filamentous hemagglutinin and pertactin for the MenACWY-CRM group versus placebo were $1.01,0.84$, and 0.82 , respectively. Predetermined non-inferiority criteria for immunological responses against all Tdap antigens were met. Co-administration of a single dose of MenACWY-CRM was well tolerated and elicited robust antibody responses against the four meningococcal serogroups, with $77 \%, 84 \%, 95 \%$ and $86 \%$ of subjects having seroprotective human complement serum bactericidal activity (titers $\geq 8$ ) against serogroups $A, C, W-135$, and $\mathrm{Y}$, respectively, one month post-vaccination.

Conclusions: Collectively, these results demonstrate that the MenACWY-CRM, Tdap and HPV4 vaccines can be administered at the same visit without compromising Tdap immune responses or increasing reactogenicity. [The study is registered with ClinicalTrials.gov, number NCT01424644].

Keywords: Meningococcal; Conjugate vaccine; Adolescents; Tdap; HPV; Neisseria meningitidis

\section{Introduction}

Infection by Neisseria meningitidis can rapidly lead to invasive meningococcal disease (IMD), resulting in death in $8-14 \%$ of cases, despite timely antibiotic treatment and supportive care [1]. Among those individuals who survive IMD, 11-19\% suffer devastating longterm sequelae, including amputations and neurological impairments. The incidence of IMD in industrialized nations is low, with estimates of 0.53 cases per 100,000 population in the United States [2].
Cases often occur sporadically, but can also occur in small clusters, with the latter becoming more common in recent years [3]. In other parts of the world, such as in Africa, IMD is associated with large epidemics, with an annual incidence greater than 1,000 cases per 100,000 population [3]. Most cases of IMD are caused by one of five pathogenic serogroups, A, B, C, W-135 or Y. Serogroup-specific disease incidence varies over time and by geographic location, thus emphasizing the need for broadly-protective vaccines.

While children younger than four years of age, particularly those under one year, remain at the greatest risk of IMD, there is a second peak in disease incidence among adolescents and young adults, with overall higher case-fatality rates among the latter age groups [2]. Social behavioral patterns that significantly increase the risk of transmission 
of meningococcus are more commonly seen in adolescents and young adults [1] and, as a corollary, these age groups exhibit the highest carriage rates [4]. In the US, the Advisory Committee on Immunization Practices (ACIP) recommends that adolescents receive a primary dose of a quadrivalent meningococcal glycoconjugate vaccine at 11 or 12 years of age, followed by a booster dose at 16 years [5].

MenACWY-CRM (Menveo ${ }^{\circ}$, Novartis Vaccines, Siena, Italy) is a meningococcal (serogroups A, C, W-135 and Y) oligosaccharide $\mathrm{CRM}_{197}$ conjugate vaccine. Unlike the predecessor unconjugated polysaccharide vaccines, glycoconjugate vaccines induce a T-cell dependent antigen response, resulting in immunological memory and longer lasting protection, and can reduce nasopharyngeal carriage, thereby promoting herd immunity [6-8]. MenACWY-CRM is licensed for use in subjects aged 2-55 years in more than 50 countries and, following the recent approval by the US Food and Drug Administration, it is the only quadrivalent meningococcal vaccine approved for use in infants two months of age and older [9]. The results of clinical trials in pediatric, adolescent and adult populations demonstrate that MenACWY-CRM is highly immunogenic and well tolerated across age groups [10-14].

In addition to the meningococcal glycoconjugate vaccine recommendations, a single booster dose of a combined tetanus, reduced diphtheria and acellular pertussis vaccine (Tdap) and three doses of a human papillomavirus (HPV) recombinant vaccine are also recommended for adolescent immunization.

An analysis of vaccination coverage in the US indicated that, while the percentage of adolescents who received all recommended vaccinations has increased in recent years, there remains a significant percentage of adolescents ( $58 \%)$ who do not receive all doses of recommended vaccines [15]. Concomitant administration of the three recommended vaccines can reduce the number of required visits to a physician and increase vaccination coverage among adolescents; however, the possibility of immunologic interference between coadministered vaccines must be evaluated.

Previous evaluations of concomitant administration of MenACWYCRM with either Tdap alone [16] or with both Tdap and HPV vaccines [17] in healthy adolescents demonstrate that MenACWY$\mathrm{CRM}$ is well tolerated and immunogenic against meningococcal serogroups A, C, W-135, and $\mathrm{Y}$ following co-administration. Moreover, concomitant administration of MenACWY-CRM did not decrease protective antibody responses to diphtheria, tetanus, or HPV. While the responses to the pertussis antigens did not meet the predefined non-inferiority criteria in the referenced studies [16,17], these findings are of unknown clinical significance, given the lack of an established serocorrelate of protection against pertussis disease. Neither of these studies included the comparison that is most relevant to current US adolescent recommendations: namely, the comparison of co-administration of MenACWY-CRM, Tdap, and HPV vaccines versus co-administration of Tdap and HPV vaccines alone.

Here we report the results of an analysis of the immune responses to a Tdap vaccine co-administered with MenACWY-CRM and a quadrivalent HPV vaccine (HPV4), as compared to Tdap coadministered with placebo and HPV4, in healthy adolescent subjects aged 10-18 years.

\section{Materials and Methods}

\section{Study Design}

This Phase IV, observer-blind, placebo-controlled, randomized study was conducted across a total of 20 sites in the United States and Italy between September, 2011 and December, 2012 (V59_40; Clinicaltrials.gov identifier: NCT01424644). This clinical study was conducted in accordance with the International Conference on Harmonization (ICH) Guidelines for Good Clinical Practice, applicable local regulations, and the Declaration of Helsinki. Prior to subject enrollment, approval of the study protocol and protocolrelated documents was obtained from an Independent Ethics Committee and written informed consent was obtained from all subjects or, for subjects under 18 years of age, parents or legal guardians.

There were two co-primary objectives for this study; one coprimary objective of this study was to assess the non-inferiority of immune responses to a Tdap vaccine when administered concomitantly with the MenACWY-CRM and HPV4 vaccines, as compared to the responses to Tdap when administered concomitantly with a placebo and HPV4. The other co-primary objective of this study was to assess the non-inferiority of immune responses to a HPV4 vaccine when administered concomitantly with MenACWY-CRM and Tdap, as compared to responses to HPV4 when co-administered with a placebo and Tdap; the results of the HPV4 immune responses are not yet available and will be published separately. Secondary objectives were to assess the immunogenicity of a single dose of MenACWYCRM when co-administered with Tdap and HPV4, and to evaluate the safety and tolerability profile of the concomitantly administered vaccines.

\section{Subjects}

A total of 801 healthy adolescents aged 10-18 years were randomized (1:1) within each study center to receive one dose each of MenACWY-CRM, Tdap and HPV4 vaccines (Men+Tdap+HPV group) or one dose each of placebo, Tdap and HPV4 vaccines (Placebo $+\mathrm{Tdap}+\mathrm{HPV}$ ) on Day 1 of the study. Subjects in both groups received two additional doses of the HPV4 vaccine, administered at two and six months after the first vaccination. Eligible study participants included those who had given their written consent/assent and if applicable, whose parents or legal guardians had given written informed consent at the time of enrollment; and those who had received childhood vaccinations against diphtheria, tetanus, and pertussis, as per local regulations, but had not yet received any adolescent vaccines against diphtheria, tetanus, or pertussis. Eligible female subjects were required to have a negative urine pregnancy test and, if sexually active, to be willing to adopt appropriate birth control measures during the study period. Subjects were excluded if they had previous disease (confirmed or suspected) caused by $N$. meningitidis, had household contact with or intimate exposure to an individual with $N$. meningitidis infection within 60 days prior to enrollment; had received any investigational or licensed vaccines containing meningococcal antigens or a prior HPV vaccination; had received or planned to receive any investigational or licensed vaccine 30 days before or after the receipt of study vaccines; had any serious acute, chronic or progressive disease, or an impaired immune system; had a history of anaphylaxis/serious vaccine reactions to any vaccine component; or had experienced significant acute infection or fever $\left(\geq 38^{\circ} \mathrm{C}\right)$ seven or three days, respectively, prior to enrollment. 


\section{Vaccines}

The three vaccines used in this study were all licensed vaccines: MenACWY-CRM (Menveo ${ }^{\circ}$, Novartis Vaccines, Siena, Italy), Tdap (Boostrix ${ }^{\oplus}$, GSK Biologicals, Rixensart, Belgium) and HPV4 (Gardasil ${ }^{\circ}$, Merck \& Co., Inc., Whitehouse Station, NJ). The MenACWY-CRM vaccine, administered as a $0.5 \mathrm{~mL}$ dose, was prepared by extemporaneous mixing, just before injection, of the lyophilized MenA-CRM component with the liquid MenCWY-CRM component. The placebo control was a sodium chloride solution (normal saline), administered as a $0.5 \mathrm{~mL}$ dose by intramuscular (IM) injection in the left deltoid. The Tdap and HPV4 vaccines were supplied as prefilled syringes and prepared according to manufacturer's instructions. The MenACWY-CRM and Tdap vaccines were administered by IM injection in the left or right deltoid, respectively; the HPV4 vaccine was administered by IM injection in the right deltoid or the anterolateral area of either thigh. All vaccines were administered by trained, unblinded site personnel.

\section{Immunogenicity analysis and endpoints}

Blood samples $(20 \mathrm{~mL})$ for immunogenicity analyses were drawn prior to the first vaccination (Visit 1; Day 1) and one month after the first vaccination (Visit 2; Month 1) (Figure 1). Antibody responses to the diphtheria, tetanus and pertussis antigens [pertussis toxin (PT), filamentous hemagglutinin (FHA) and pertactin (PRN)] were measured by enzyme-linked immunosorbent assay (ELISA). Serological analyses against the Tdap antigens were performed by Pharmaceutical Product Development, LLC (PPD; Wayne, Pennsylvania). Anti-diphtheria and anti-tetanus antitoxin responses were expressed as geometric mean concentrations (GMCs) and the percentage of subjects with antibody levels $\geq 1.0 \mathrm{IU} / \mathrm{mL}$, which are correlates of protection. Anti-pertussis antibody responses were expressed as GMCs and percentages of subjects with seroresponse for each of the three antigens. Seroresponse for anti-PT, anti-FHA and anti-PRN antibodies was defined as follows: for subjects with a prevaccination antibody concentration below the lower limit of quantitation (LLQ) (equal to $0.9,2.9$ and $3.0 \mathrm{IU} / \mathrm{mL}$ for PT, FHA and $\mathrm{PRN}$, respectively), a post-vaccination antibody concentration of at least four times the LLQ; for subjects with a pre-vaccination antibody concentration $\geq \mathrm{LLQ}$, an antibody concentration of at least four times the pre-vaccination concentration.

Immunogenicity of the MenACWY-CRM vaccine was assessed by serum bactericidal assay using human complement (hSBA) against $N$. meningitidis serogroups $\mathrm{A}, \mathrm{C}, \mathrm{W}-135$ and $\mathrm{Y}$ and expressed as percentage of subjects with seroresponse, percentage of subjects with hSBA titers $\geq 8$ [18] and hSBA geometric mean titers (GMTs). Seroresponse was defined by an increase from pre-vaccination hSBA titers $<4$ to post vaccination hSBA titers $\geq 8$, or at least a four-fold increase in hSBA titers for subjects with pre-vaccination titers $\geq 4$. The serological analyses for MenACWY-CRM were performed at Novartis Vaccines, Clinical Serology Laboratory in Marburg, Germany.

\section{Safety analysis}

Subjects were observed for 30 minutes after vaccination for any immediate reactions by blinded personnel. Subjects (or their parents/ guardians, when applicable) were provided with diary cards to record solicited local and systemic reactions up to seven days after the Day 1 vaccination. Solicited local reactions, evaluated only for the MenACWY-CRM and placebo injection sites, included pain at injection site, erythema and induration. Solicited systemic reactions included chills, nausea, malaise, myalgia, arthralgia, and headache, rash and fever $\left(\geq 38.0^{\circ} \mathrm{C}\right)$. The reactions were described by the subject or parent/guardian as mild, moderate, or severe. Serious adverse events (SAEs), medically attended adverse events (AEs) and other unsolicited AEs (including new onset of chronic illness) were collected from Day 1 to the end of the study and were judged by the investigator as possibly related, probably related or not related to study vaccination. All subjects who received at least one vaccination and provided any safety data were considered evaluable for the safety analyses.

\section{Statistical analysis}

Assuming $10 \%$ early terminated or non-eligible for analysis subjects, approximately 800 subjects were planned to be enrolled in the study (400 per vaccine group) in order to obtain 360 evaluable subjects in each group. There were nine non-inferiority hypotheses underlying the co-primary objectives (five Tdap and four HPV4) that were tested in a stepwise approach, starting with the five Tdap non-inferiority hypotheses. Due to the pre-specified ordering of the two co-primary objectives, no alpha adjustment was required. The study sample size afforded at least $98 \%$ power to reject each of the nine individual hypotheses. Therefore, the overall power to reject all nine hypotheses underlying the two co-primary objectives and conclude noninferiority of Tdap and HPV4 immune responses to MenACWY $+\mathrm{Tdap}+\mathrm{HPV}$ versus Placebo+Tdap+HPV was at least $83 \%$ $\left(0.98^{9} \times 100 \%\right)$.

The Tdap immunogenicity analyses were conducted on the Tdap per protocol (PP) population $[\mathrm{n}=758(95 \%)]$, which included subjects who correctly received the relevant vaccinations, provided evaluable serum samples on Day 1 and at one month-post vaccination and had no major protocol deviations. Non-inferiority criteria for antidiphtheria and anti-tetanus antibodies were as follows: the lower limit (LL) of the two-sided $95 \%$ confidence interval (CI) on the difference between the groups $[(\mathrm{Men}+\mathrm{Tdap}+\mathrm{HPV})$ minus (Placebo+Tdap $+\mathrm{HPV})]$ in the percentage of subjects with anti-tetanus and antidiphtheria toxin antibody levels $\geq 1.0 \mathrm{IU} / \mathrm{mL}$ must be greater than $-10 \%$ for each antigen. Non-inferiority criteria for pertussis antigens were as follows: the LL of the two-sided $95 \%$ CI of the inter-group ratio (Men+Tdap+HPV to Placebo+Tdap+HPV) of GMCs for each pertussis antigen (PT, FHA, PRN) must be greater than 0.5. The twosided $95 \%$ CIs for group differences in diphtheria and tetanus response rates were constructed using the method of Mee [19]. The group GMC ratios for the anti-pertussis responses and their associated 2-sided $95 \%$ CIs were computed by exponentiating (base ${ }_{10}$ ) the corresponding vaccine group difference in least square means of the $\log 10$ transformed concentrations and the associated 95\% CI for the difference obtained from an ANOVA model having factors for vaccine group and study center. Data analyses were performed using $\mathrm{SAS}^{\bullet}$ version 9.1.

The analyses of immune responses against $N$. meningitidis serogroups A, C, W-135 and Y were performed on the MenACWYCRM PP population, which included all eligible subjects from the Men $+\mathrm{Tdap}+\mathrm{HPV}$ group $(\mathrm{N}=372)$ and a randomly selected subset of subjects from the Placebo+Tdap+HPV group $(\mathrm{N}=100)$.

Safety analyses were primarily descriptive. Differences between the vaccine groups in the frequency of solicited reactions reported after the first vaccination were analyzed with Pearson's chi-square test, or Fisher's Exact test, in the event of small expected cell sizes. 
Citation: Gasparini R, Johnston W, Conversano M, Garscadden A, Alexanderian D, et al. (2014) Immunogenicity and Safety of Combined Tetanus, Reduced Diphtheria, Acellular Pertussis Vaccine when Co-Administered with Quadrivalent Meningococcal Conjugate and Human Papillomavirus Vaccines in Healthy Adolescents. J Vaccines Vaccin 5: 231. doi:10.4172/2157-7560.1000231

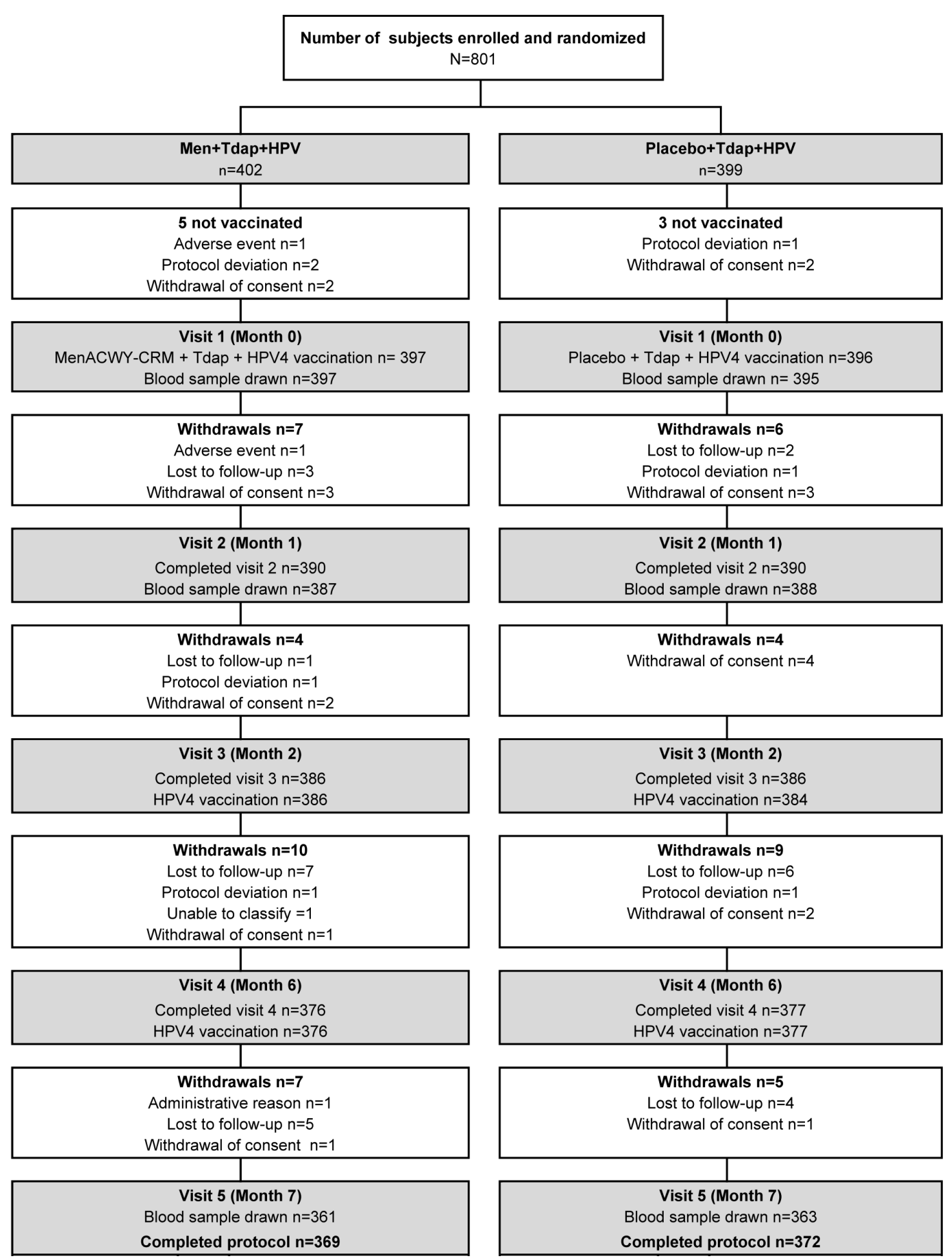

Figure 1: Subject disposition flowchart. 
Citation: Gasparini R, Johnston W, Conversano M, Garscadden A, Alexanderian D, et al. (2014) Immunogenicity and Safety of Combined Tetanus, Reduced Diphtheria, Acellular Pertussis Vaccine when Co-Administered with Quadrivalent Meningococcal Conjugate and Human Papillomavirus Vaccines in Healthy Adolescents. J Vaccines Vaccin 5: 231. doi:10.4172/2157-7560.1000231

Page 5 of 10

\section{Results}

\section{Enrollment, study flow and demographics}

Among 801 enrolled subjects, 793 (99\%) were vaccinated on Day 1 and 741 (93\%) completed the study. Reasons for premature withdrawal included: lost to follow up $(n=28)$, withdrawal of consent $(n=21)$, protocol deviations $(n=7)$, administrative reasons $(n=1)$, and unable to classify $(n=1)$. Additionally, two subjects from the Men + Tdap+HPV group withdrew due to an adverse event (syncope, $n=1$; pyrexia and headache, $n=1$ ), of which only one (with pyrexia and headache) had received a study vaccination.

In the enrolled population, demographic parameters, such as age, sex and race, were generally similar across the study groups (Table 1). The average age of the subjects at study entry was $11.9( \pm 1.6)$ years and the majority of the subjects were male $(60 \%)$. Other baseline characteristics such as mean weight, height and body mass index were well balanced across the study groups.

\begin{tabular}{|c|c|c|c|}
\hline & Men+Tdap+HPV & Placebo+Tdap+HPV & Total \\
\hline & $n=402$ & $n=399$ & $\mathrm{~N}=801$ \\
\hline Age (Yrs) & $11.9 \pm 1.7$ & $11.8 \pm 1.5$ & $11.9 \pm 1.6$ \\
\hline \multicolumn{4}{|l|}{ Sex } \\
\hline Male & $233(58 \%)$ & $244(61 \%)$ & $477(60 \%)$ \\
\hline Female & $169(42 \%)$ & $155(39 \%)$ & $324(40 \%)$ \\
\hline \multicolumn{4}{|l|}{ Race } \\
\hline Asian & $5(1 \%)$ & $7(2 \%)$ & $12(1 \%)$ \\
\hline $\begin{array}{l}\text { Black, } \\
\text { Hispanic }\end{array}$ & $39(10 \%)$ & $36(9 \%)$ & $75(9 \%)$ \\
\hline $\begin{array}{l}\text { White, } \\
\text { Hispanic }\end{array}$ & $319(79 \%)$ & $310(78 \%)$ & $629(79 \%)$ \\
\hline $\begin{array}{l}\text { Native American/ } \\
\text { Alaskan }\end{array}$ & $1(<1 \%)$ & $2(<1 \%)$ & $3(<1 \%)$ \\
\hline Pacific/Hawaii & 0 & $2(<1 \%)$ & $2(<1 \%)$ \\
\hline Other & $38(9 \%)$ & $42(11 \%)$ & $80(10 \%)$ \\
\hline Weight (kg & $50.4 \pm 15.7$ & $50.6 \pm 15.3$ & $50.5 \pm 15.5$ \\
\hline
\end{tabular}

\begin{tabular}{|c|c|c|c|c|c|c|}
\hline \multirow[t]{2}{*}{ Antigen } & \multirow[t]{2}{*}{ Timing } & \multicolumn{2}{|r|}{ Men+Tdap+HPV } & \multicolumn{2}{|c|}{ Placebo+Tdap+HPV } & \multirow[t]{2}{*}{ Vaccine Group Differences / Ratiosł } \\
\hline & & $\mathrm{n}$ & $\%$ of subjects/GMC & $\mathrm{n}$ & $\%$ of subjects/GMC & \\
\hline & & & $\% \geq 1.0 \mathrm{IU} / \mathrm{mL}(95 \% \mathrm{Cl})$ & & $\% \geq 1.0 \mathrm{IU} / \mathrm{mL}(95 \% \mathrm{Cl})$ & $\begin{array}{c}\text { Men+Tdap+HPV minus Placebo+Tdap } \\
+ \text { HPV }(95 \% \mathrm{Cl})\end{array}$ \\
\hline \multicolumn{7}{|l|}{ Diphtheria } \\
\hline & Baseline & 375 & $5(3,8)$ & 380 & $3(2,5)$ & $2(-1,5)$ \\
\hline & Month 1 & 376 & $95(93,97)$ & 382 & $82(78,86)$ & $13(9,17)$ \\
\hline \multicolumn{7}{|l|}{ Tetanus } \\
\hline & Baseline & 375 & $28(24,33)$ & 380 & $28(23,32)$ & $1(-6,7)$ \\
\hline & Month 1 & 376 & $99(97,100)$ & 382 & $98(97,99)$ & $0(-2,2)$ \\
\hline
\end{tabular}

Tdap immunogenicity: One month post-Tdap vaccination, the percentages of subjects with seroprotective anti-diphtheria or antitetanus antibody concentrations $(\geq 1.0 \mathrm{IU} / \mathrm{mL})$ were high in both vaccine groups. While there was a greater proportion of subjects with seroprotective anti-diphtheria antibody concentrations in the Men + Tdap+HPV group as compared to the Placebo+Tdap+HPV group, ( $95 \%$ vs. $82 \%$, respectively), the percentage of subjects with seroprotective anti-tetanus antibody concentrations was similar between groups (Men+Tdap+HPV: 99\%; Placebo+Tdap+HPV: 98\%) (Table 2). The LL of the two-sided 95\% CI for the difference in the proportion of subjects with seroprotective anti-diphtheria and antitetanus antibody concentrations between the study groups (Men $+\mathrm{Tdap}+\mathrm{HPV}$ minus Placebo+Tdap+HPV) at one month following vaccination was $9 \%$ and $-2 \%$, respectively (Table 2 ), satisfying noninferiority criteria for the diphtheria and tetanus antigens.

Immune responses against the three pertussis antigens were similar one month following co-administration of Tdap with MenACWYCRM and HPV or a placebo and HPV, with increases relative to baseline in the GMCs of antibodies to pertussis toxin (PT), filamentous hemagglutinin (FHA) and pertactin (PRN) of 9.2 to 11fold, 8.4 to 11 fold, and 16 to 19 -fold, respectively, across the two groups (Table 2). Non-inferiority of antibody responses to each of the three pertussis antigens was also demonstrated at one month postvaccination when Tdap was co-administered with MenACWY-CRM and HPV4 vaccines, as compared to when Tdap was administered with a placebo and the HPV4 vaccine. Specifically, the LL of the two-sided 95\% CI for the group ratio (Men+Tdap+HPV to Placebo+Tdap+HPV) of the GMCs of antibodies to PT, FHA and PRN were all greater than 0.5 (PT, 0.89; FHA, 0.76; PRN, 0.72; Table 2). 
Citation: Gasparini R, Johnston W, Conversano M, Garscadden A, Alexanderian D, et al. (2014) Immunogenicity and Safety of Combined Tetanus, Reduced Diphtheria, Acellular Pertussis Vaccine when Co-Administered with Quadrivalent Meningococcal Conjugate and Human Papillomavirus Vaccines in Healthy Adolescents. J Vaccines Vaccin 5: 231. doi:10.4172/2157-7560.1000231

Page 6 of 10

\begin{tabular}{|l|l|c|c|c|c|c|}
\hline Pertussis antigen & & & GMC (IU/ml\#) (95\% Cl) & & GMC (95\% Cl) & $\begin{array}{c}\text { Ratio of Men+Tdap+HPV to Placebo } \\
+ \text { Tdap+HPV (95\% Cl) }\end{array}$ \\
\hline \multirow{2}{*}{ PT } & Baseline & 375 & $4.77(4.11,5.53)$ & 380 & $4.16(3.59,4.82)$ & $1.15(0.95,1.39)$ \\
\cline { 2 - 7 } & Month 1 & 376 & $44(40,48)$ & 382 & $44(40,48)$ & $1.01(0.89,1.14)$ \\
\hline \multirow{2}{*}{ FHA } & Baseline & 375 & $24(21,27)$ & 380 & $21(18,23)$ & $1.15(0.98,1.34)$ \\
\cline { 2 - 7 } & Month 1 & 376 & $202(187,218)$ & 382 & $240(222,259)$ & $0.84(0.76,0.93)$ \\
\hline Pertactin & Baseline & 375 & $20(18,23)$ & 380 & $21(18,24)$ & $0.99(0.83,1.18)$ \\
\cline { 2 - 7 } & Month 1 & 376 & $330(300,363)$ & 382 & $403(367,443)$ & $0.82(0.72,0.93)$ \\
\hline
\end{tabular}

Table 2: Differences in Tdap antigen-specific immune responses between the Men+Tdap+HPV and Placebo+Tdap+HPV groups. Percentages (95\% confidence intervals) of subjects with seroprotective anti-diphtheria toxin and anti-tetanus toxin antibody levels and GMCs (95\% confidence intervals) of antibodies against pertussis antigens, at baseline and one month after Tdap vaccination. 拴old values indicate that the non-inferiority criteria were met. \#GMCs are shown as ELISA units/ml

GMCs for anti-diphtheria and anti-tetanus antibodies are presented in Figure 2. While the GMCs of anti-diphtheria antibodies were similar in both vaccine groups prior to vaccination, there was a greater increase in the GMCs one month after vaccination for the Men+Tdap + HPV group (39-fold) as compared to the Placebo+Tdap+HPV group (15-fold) (Figure 2A). GMCs for the anti-tetanus antibodies were similar between groups both prior to vaccination and one month postvaccination, with increases of 12 - and 15-fold in the Men+Tdap+HPV group and Placebo+Tdap+HPV group, respectively (Figure 2A).

Additional analyses for antibody responses to the pertussis antigens did not show any differences between the Men+Tdap+HPV and
Placebo+Tdap+HPV groups for the percentage of subjects in each group achieving seroresponse for anti-PT antibodies (Men+Tdap $+\mathrm{HPV}, 80 \%$; Placebo+Tdap+HPV, 84\%) and anti-PRN antibodies (Men+Tdap+HPV, 88\%; Placebo+Tdap+HPV, 92\%) at one month post-vaccination; whereas the percentage of subjects achieving seroresponse for anti-FHA antibodies was lower in the Men+Tdap $+\mathrm{HPV}$ group (72\%) than the Placebo+Tdap+HPV group (81\%) (Figure 2B).
2A

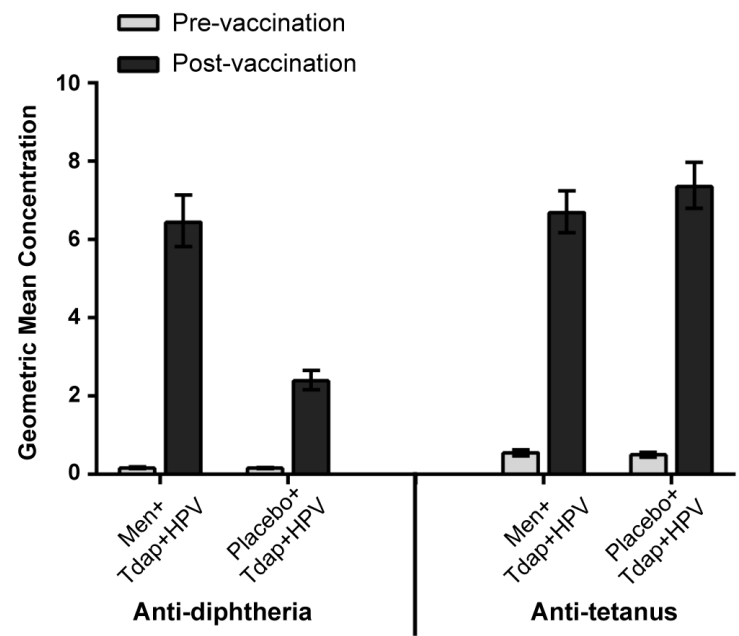

2B

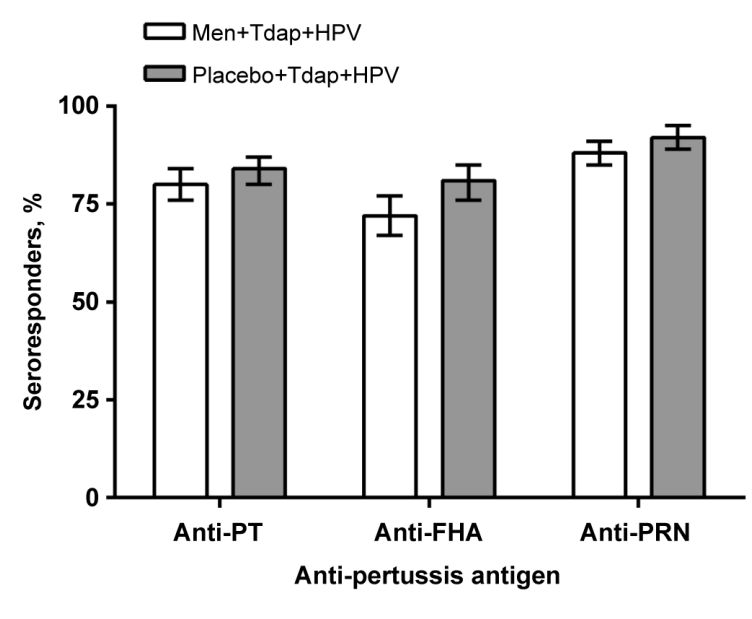

Figure 2: Tdap immunogenicity endpoints at one month post-Tdap-vaccination. (A) Geometric mean concentrations of anti-diphtheria and anti-tetanus antibodies at baseline and one month post-vaccination, by group. (B) The percentage of subjects with seroresponse to the pertussis antigens, pertussis toxin (PT), filamentous hemagglutinin (FHA), and pertactin (PRN), by group.

MenACWY-CRM Immunogenicity: The majority of subjects in both the Men+Tdap+HPV group and the Placebo+Tdap+HPV group were seronegative (hSBA titers $<4)$ at baseline for serogroups A $(95 \%$ vs. $96 \%$, respectively), C (57\% vs. $55 \%)$ and Y ( $62 \%$ vs. $72 \%)$; whereas, the percentages of subjects who were seronegative for serogroup
$\mathrm{W}-135$ prior to vaccination were overall lower (Men+Tdap+HPV group, 34\%; Placebo+Tdap+HPV group, 32\%). Robust immune responses against all four serogroups were evident at one month following co-administration of a single dose of MenACWY-CRM with the Tdap and HPV4 vaccines, with $77 \%, 84 \%, 95 \%$, and $86 \%$ of 
Citation: Gasparini R, Johnston W, Conversano M, Garscadden A, Alexanderian D, et al. (2014) Immunogenicity and Safety of Combined Tetanus, Reduced Diphtheria, Acellular Pertussis Vaccine when Co-Administered with Quadrivalent Meningococcal Conjugate and Human Papillomavirus Vaccines in Healthy Adolescents. J Vaccines Vaccin 5: 231. doi:10.4172/2157-7560.1000231

Page 7 of 10

subjects in the Men+Tdap+HPV group achieving hSBA titers $\geq 8$ against meningococcal serogroups $\mathrm{A}, \mathrm{C}, \mathrm{W}-135$ and $\mathrm{Y}$, respectively (Table 3). As expected, at one month post-vaccination, the percentages of subjects in the Placebo+Tdap+HPV group with hSBA titers $\geq 8$ against the four meningococcal serogroups were similar to baseline. The percentages of subjects achieving seroresponse were high for serogroups A, C and Y $(75 \%, 69 \%$, and $69 \%$, respectively); whereas, the percentage of subjects achieving seroresponse for serogroup W-135 was lower (49\%)). A lower seroresponse to serogroup W-135 reflects the high percentage of seropositive subjects $(>65 \%)$ at baseline. In contrast, following co-administration of the placebo with the Tdap and HPV4 vaccines, only $1-5 \%$ of subjects showed seroresponse against each individual serogroup.

In the Men+Tdap+HPV group, at one month post-vaccination the GMT increases relative to baseline were higher against serogroups A (16-fold), C (13-fold), and Y (12-fold), as compared to the increases against serogroup $\mathrm{W}-135$ (5-fold; Table 3); the lower relative increase in GMTs against serogroup W-135 reflect the higher baseline hSBA titers for this serogroup across both groups. As anticipated, there was no change in GMTs from baseline to one month post-vaccination in the Placebo+Tdap+HPV group.

\begin{tabular}{|c|c|c|c|c|}
\hline \multirow[t]{2}{*}{ Serogroup } & \multicolumn{2}{|c|}{ Men+Tdap+HPV } & \multicolumn{2}{|c|}{ Placebo+Tdap+HPV } \\
\hline & $\mathbf{n}$ & GMT / $\%$ of subjects & $\mathbf{n}$ & GMT / $\%$ of subjects \\
\hline A & 371 & & 99 & \\
\hline \multicolumn{5}{|l|}{ GMTs } \\
\hline Baseline & & $2.2(2.1,2.3)$ & & $2.1(2.0,2.3)$ \\
\hline 1 month post-vaccination & & $35(29,42)$ & & $2.1(2.0,2.3)$ \\
\hline hSBA titer $\geq 8, \%$ & & $77(72,81)$ & & $2(0,7)$ \\
\hline Seroresponders, \% & & $75(70,80)$ & & $1(0,5)$ \\
\hline C & 370 & & 97 & \\
\hline \multicolumn{5}{|l|}{ GMTs } \\
\hline Baseline & & $4.4(4.0,4.9)$ & & $3.9(3.3,4.6)$ \\
\hline 1 month post-vaccination & & $59(48,73)$ & & $3.9(3.3,4.7)$ \\
\hline hSBA titer $\geq 8, \%$ & & $84(80,88)$ & & $21(13,30)$ \\
\hline Seroresponders, \% & & $69(64,74)$ & & $2(0,7)$ \\
\hline W-135 & 369 & & 96 & \\
\hline \multicolumn{5}{|l|}{ GMTs } \\
\hline Baseline & & $12(10,14)$ & & $12(9.1,16)$ \\
\hline 1 month post-vaccination & & $61(53,69)$ & & $12(9.3,17)$ \\
\hline hSBA titer $\geq 8, \%$ & & $95(93,97)$ & & $67(56,76)$ \\
\hline Seroresponders, \% & & $49(44,54)$ & & $5(2,12)$ \\
\hline $\mathbf{Y}$ & 369 & & 97 & \\
\hline \multicolumn{5}{|l|}{ GMT } \\
\hline Baseline & & $4.1(3.7,4.6)$ & & $3.5(2.8,4.3)$ \\
\hline 1 month post-vaccination & & $48(40,58)$ & & $3.5(2.9,4.3)$ \\
\hline hSBA titer $\geq 8, \%$ & & $86(82,89)$ & & $22(14,31)$ \\
\hline Seroresponders, \% & & $69(64,74)$ & & $3(1,9)$ \\
\hline
\end{tabular}

Table 3: Immunogenicity endpoints for meningococcal serogroups A, C, W-135 and Y responses. hSBA GMTs, percentage of subjects with hSBA titer $\geq 8$, and percentage of seroresponders against serogroups $A, C, W-135$, and $\mathrm{Y}$, at baseline (GMTs only) and one month post vaccination, by group. 
Citation: Gasparini R, Johnston W, Conversano M, Garscadden A, Alexanderian D, et al. (2014) Immunogenicity and Safety of Combined Tetanus, Reduced Diphtheria, Acellular Pertussis Vaccine when Co-Administered with Quadrivalent Meningococcal Conjugate and Human Papillomavirus Vaccines in Healthy Adolescents. J Vaccines Vaccin 5: 231. doi:10.4172/2157-7560.1000231

Page 8 of 10

\section{Reactogenicity and Safety}

Among 801 subjects in the enrolled population, 793 (99\%) subjects were exposed to the study vaccines, all of whom provided postvaccination safety data and were included in the safety analyses. Excluding those individuals for whom data on solicited reactions were not available, 774 (97\%) subjects were included in reactogenicity analyses; the percentages of subjects with any reported solicited reaction within seven days of the first vaccination was $72 \%$ for the Men+Tdap+HPV group and 64\% for the Placebo+Tdap+HPV group. Overall, the reported rates of both local and systemic solicited reactions were higher in the Men+Tdap+HPV group than the Placebo + Tdap+HPV group (local reactions: $54 \%$ vs. $43 \%$; systemic reactions: $53 \%$ vs. $46 \%$, respectively). Other solicited indicators of reactogenicity (i.e., subjects stayed at home due to any reaction or used analgesics or antipyretics) were reported in similar percentages of subjects for both groups (Men+Tdap+HPV group, 23\%; Placebo+Tdap+HPV group, $21 \%)$.
The most commonly reported solicited local reaction (at the MenACWY-CRM or placebo injection site) within seven days following vaccination was pain at the injection site, which was reported by similar percentages of subjects in both groups (Men+Tdap $+\mathrm{HPV}, 35 \%$ versus Placebo+Tdap+HPV, 41\%), followed by erythema $(17 \%$ versus $7 \% ; \mathrm{p}<0.001)$ and induration $(16 \%$ versus $10 \% ; \mathrm{p}=0.011)$ (Figure $3 \mathrm{~A}$ ). Severe solicited local reactions were reported by up to $1 \%$ of subjects across both vaccine groups.

The most commonly reported solicited systemic reaction within seven days following vaccination was myalgia (26-30\%), followed by headache (25-29\%), chills (13-15\%) and malaise (11-15\%), with no statistically significant differences between groups in the frequencies of each solicited systemic reaction (Figure 3B). Severe solicited systemic reactions or fever were reported in no more than $1 \%$ or $2 \%$ of subjects, respectively, in either vaccine group.

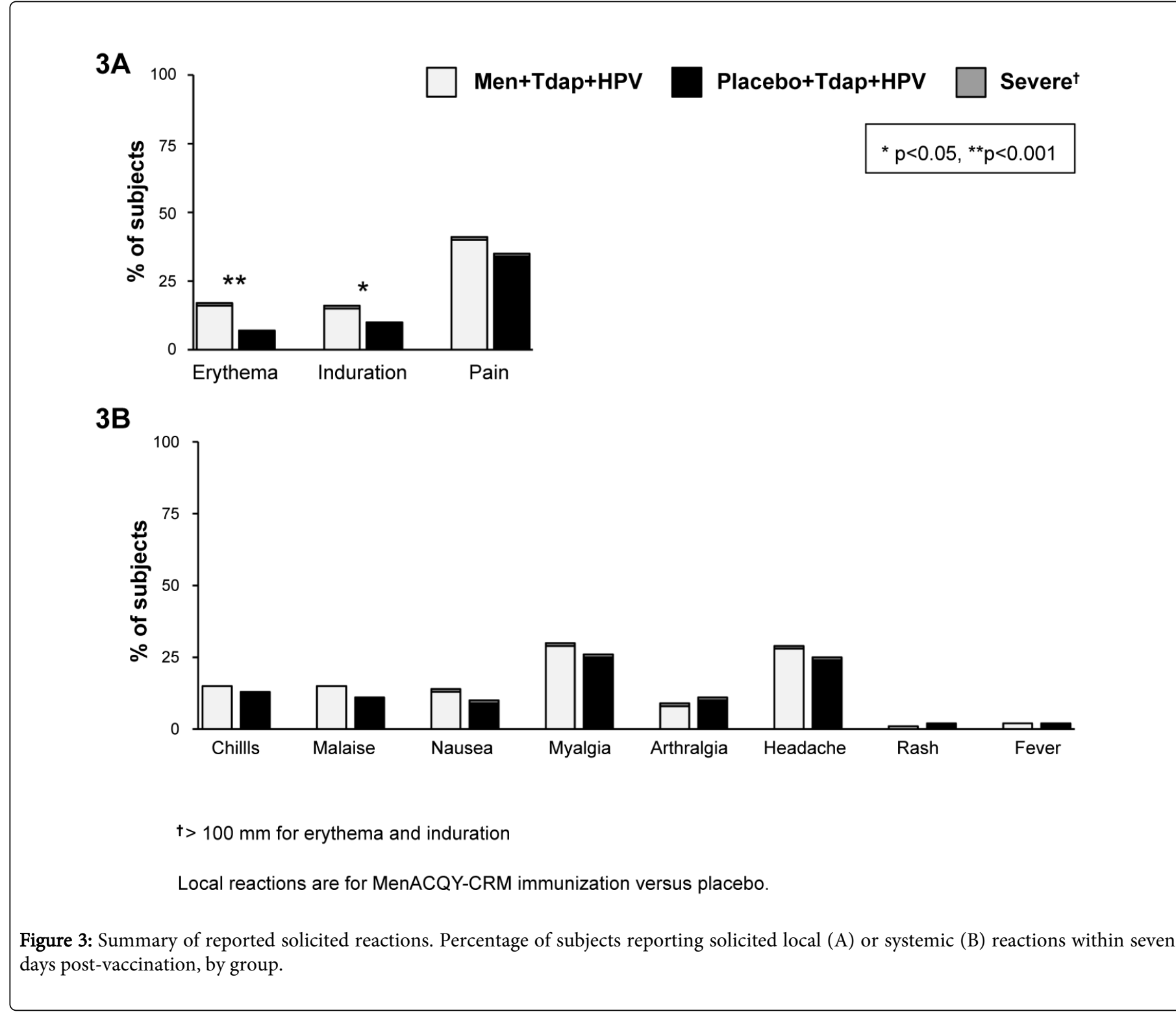


Unsolicited AEs were reported by similar percentages of subjects in the Men+Tdap+HPV and Placebo+Tdap+HPV groups (51\% versus $50 \%$, respectively), with the most common unsolicited AEs being injection site pain $($ Men+Tdap+HPV, 7\% vs. Placebo+Tdap+HPV, $6 \%$ ) and pharyngitis (Men+Tdap+HPV, 6\% vs. Placebo+Tdap+HPV, $5 \%)$. The frequencies of diagnoses of new onset chronic disease were similar between the Men+Tdap+HPV group (6\%) and the Placebo + Tdap+HPV group (7\%). The most frequently reported chronic conditions were seasonal allergies and minor dermatology conditions, such as acne and atopic dermatitis, consistent with expected patterns for this age group.

A total of seven subjects reported nine SAEs during the entire study period, with hematuria, abdominal adhesion, small intestinal obstruction, aggression, hypothyroidism and type 1 diabetes mellitus reported by subjects in the Men+Tdap+HPV group and peritonsillar abscess, accidental overdose and affective disorder reported in the Placebo+Tdap+HPV group. With the exception of the subject with affective disorder, all subjects recovered from the SAEs (or recovered from acute manifestations of the SAEs, in the case of the subject with hypothyroidism and type 1 diabetes mellitus), and none of the SAEs were considered to be related to the study vaccine. There were no deaths reported in this study.

\section{Discussion}

Adolescents are at increased risk of vaccine-preventable diseases, due to waning protective immunity conferred by childhood vaccination against pertussis, as well as social behaviors that increase their exposure to meningococcus, Bordetella pertussis, and HPV [20]. While immunization compliance among adolescents is not very well monitored in most countries, estimates of vaccine coverage in this age group in the US indicate that compliance is lower than that observed for vaccinations recommended for infants and toddlers [15]. Lower immunization compliance among adolescents is attributed, at least in part, to infrequent healthcare visits and the resulting missed opportunity for vaccination $[20,21]$. Concomitant administration of recommended vaccines can increase compliance in adolescents substantially, provided that immunologic interference between vaccines does not occur. In particular, the results of an analysis on adolescent vaccination coverage in the US indicate that simultaneous administration of indicated vaccines could increase coverage for Tdap and MenACWY vaccines from $\geq 54 \%$ to $>80 \%$, and for the first dose of a HPV vaccine from $44 \%$ to $74 \%$ [15].

In the present study, we demonstrate that following coadministration of the MenACWY-CRM, Tdap, and HPV4 vaccines, immune responses to all five Tdap antigens were non-inferior to those following co-administration of a placebo with the Tdap and HPV4 vaccines. In contrast to previous studies examining co-administration of MenACWY-CRM and Tdap [16,17], non-inferiority criteria were met for all three pertussis antigens tested.

In the earlier studies, the direct comparators for Tdap immune responses were either Tdap alone [17] or Tdap administered with a placebo [16]. In contrast, the direct comparator group of the present study received a placebo with both Tdap and HPV4. The differences in the responses to the pertussis antigens between the studies could be attributed to the different control groups or to other factors, such as geographic location or variability in the immune assays used to assess these responses.
In this study, we found that antibody concentrations to the diphtheria toxoid one month after vaccination were substantially higher in the Men+Tdap+HPV group than the Placebo+Tdap+HPV group. Similar augmentation of anti-diphtheria responses in adolescents following co-administration of MenACWY-CRM with Tdap has been reported previously $[16,17]$ and is likely attributed to the use of $\mathrm{CRM}_{197}$, a nontoxic mutant of diphtheria toxin, as the carrier protein in MenACWY-CRM; however, the clinical relevance of this augmented response is not known.

We also found that co-administration of MenACWY-CRM with Tdap and HPV4 vaccines induced robust immune responses to $N$. meningitidis serogroups A, C, W-135, and Y; the high percentages of subjects with hSBA titers $\geq 8$ against the four serogroups (77-95\%) were similar to those observed previously following co-administration of MenACWY-CRM with Tdap and HPV4 vaccines [17] or MenACWY-CRM with Tdap [16] in adolescents. In addition, the responses against serogroups $\mathrm{A}, \mathrm{C}, \mathrm{W}-135, \mathrm{Y}$ following concomitant administration of MenACWY-CRM, Tdap and HPV4 vaccines observed in this study were similar to those observed in adolescents following administration of MenACWY-CRM alone [12].

The relatively low overall seroresponse rate to serogroup $\mathrm{W}-135$ in the Men+Tdap+HPV group is likely attributable to the high percentage of subjects (66\%) with hSBA titers $\geq 4$ against this serogroup prior to vaccination. The high percentage of baseline serogroup W-135 seropositivity observed in this study has been described elsewhere $[12,17]$, and could be attributed to cross-reactive antibodies, or could potentially reflect the high rates of asymptomatic nasopharyngeal carriage observed among adolescents and young adults [4].

In the present study, the safety profile of the MenACWY-CRM vaccine concomitantly administered with Tdap and HPV4 vaccines, including the rates of any AEs, possibly related AEs, medicallyattended AEs and new onset of chronic disease, was similar to that of the placebo, co-administered with the Tdap and HPV4 vaccines. The pattern of individual unsolicited AEs reported was typical in adolescents and similar in both study groups.

Reports of any solicited reaction were overall higher in the Men $+\mathrm{Tdap}+\mathrm{HPV}$ group as compared to the Placebo+Tdap+HPV group; however, among all reported reactions, statistically significant differences between groups were only observed for the rates of erythema and induration, which were assessed at the injection site for MenACWY-CRM and placebo only. For all local and systemic reactions, most were mild to moderate in nature, with $\leq 1 \%$ of subjects reporting severe solicited reactions. Overall, the frequencies of these reactions were similar to those observed previously in adolescents who had received MenACWY-CRM either alone [12] or co-administered with Tdap and HPV4 vaccines [17].

Collectively, the results of this study demonstrate that MenACWYCRM co-administered with Tdap and HPV4 vaccines does not interfere with immune responses against the tetanus, diphtheria, and pertussis antigens in Tdap. Moreover, the results demonstrate that MenACWY-CRM co-administered with Tdap and HPV4 vaccines elicits highly immunogenic responses against meningococcal serogroups A, C, W-135 and Y, and has an acceptable reactogenicity profile. These studies support the concomitant use of MenACWYCRM with other recommended adolescent vaccines, thereby increasing the likelihood of vaccine compliance among this age group. 
Citation: Gasparini R, Johnston W, Conversano M, Garscadden A, Alexanderian D, et al. (2014) Immunogenicity and Safety of Combined Tetanus, Reduced Diphtheria, Acellular Pertussis Vaccine when Co-Administered with Quadrivalent Meningococcal Conjugate and Human Papillomavirus Vaccines in Healthy Adolescents. J Vaccines Vaccin 5: 231. doi:10.4172/2157-7560.1000231

Page 10 of 10

\section{Acknowledgements}

The authors are grateful to all subjects, and their families, who participated in the clinical trial. The authors thank Novartis Vaccines personnel: Barbara Clough, David Cooke, Conor Knightly, Caitlin McCourt, Tatjana Odrljin MD, Michelangelo Barone, Laura Lulli, Tiziana Pungì and Eva Leeuwenhoek (Clinical), as well as Sonja Haegele (Serology), Freekje Bär (Data Management), Darren Kelly (Data analysis) and Ellen Ypma (Biostatistics). Medical writing and editorial assistance in the preparation of this manuscript was provided by Shivani Vadapalli, PhD and Jessica Tyler, PhD (both Novartis Vaccines). Novartis Vaccines and Diagnostics, Inc. provided financial support for the conduct of the research including study design, data collection and analysis.

\section{Investigators}

United States: Drs. Wilson Andrews, Richard Ohnmacht, Robert Stacks, Martin Schear, Aftab Naz, Julie Shepard, Brandon Essink, Robert Saniuk, Thomas McKnight, Scott Strahlman, Ruffin Franklin, Edward Zissman, Jennifer Kay, and Paul Bernhardson. Italy: Professors Gianni Bona, Giulia Genoni, Agata Bizzocchi, Miriam Gatto, Laura Panigati, Roberta Ricotti, Sandra Esposito, Rocco Iudici, Sesilja Vyshka, Daniela Amicizia, Donatella Panatto, Augusto Giorgino, Carlo Calamai, and Luigi Mastronuzzi, as well as Valentina Pirelli and Luciana Marchianò.

\section{References}

1. Pace D, Pollard AJ (2012) Meningococcal disease: Clinical presentation and sequelae. Vaccine 30 Suppl 2: B3-9.

2. Cohn AC, MacNeil JR, Harrison LH, Hatcher C, Theodore J, et al. (2010) Changes in Neisseria meningitidis disease epidemiology in the United States, 1998-2007: Implications for prevention of meningococcal disease. Clin Infect Dis 50: 184-91.

3. Harrison LH, Trotter CL, Ramsay ME (2009) Global epidemiology of meningococcal disease. Vaccine 27 Suppl 2: B51-63.

4. Claus H, Maiden MC, Wilson DJ, McCarthy ND, Jolley KA, et al. (2005) Genetic analysis of meningococci carried by children and young adults. J Infect Dis 191: 1263-1271.

5. Centers for Disease Control and Prevention (CDC) (2011) Updated recommendations for use of meningococcal conjugate vaccines -.Advisory Committee on Immunization Practices (ACIP), 2010. MMWR Morb Mortal Wkly Rep 60: 72-76.

6. Kelly DF, Snape MD, Clutterbuck EA, Green S, Snowden C, et al. (2006) CRM197-conjugated serogroup C meningococcal capsular polysaccharide, but not the native polysaccharide, induces persistent antigen-specific memory B cells. Blood 108: 2642-2647.

7. Trotter CL, Maiden MC (2009) Meningococcal vaccines and herd immunity: lessons learned from serogroup $C$ conjugate vaccination programs. Expert Rev Vaccines 8: 851-861.
8. Daugla DM, Gami JP, Gamougam K, Naibei N, Mbainadji L, et al. (2014) Effect of a serogroup A meningococcal conjugate vaccine (PsA-TT) on serogroup A meningococcal meningitis and carriage in Chad: a community study [corrected]. Lancet 383: 40-47.

9. United States Food and Drug Administration (2013) August 1, 2013 Approval Letter - Menveo.

10. Klein NP, Reisinger KS, Johnston W, Odrljin T, Gill CJ, et al. (2012) Safety and immunogenicity of a novel quadrivalent meningococcal CRM-conjugate vaccine given concomitantly with routine vaccinations in infants. Pediatr Infect Dis J 31: 64-71.

11. Black S, Klein NP, Shah J, Bedell L, Karsten A, et al. (2010) Immunogenicity and tolerability of a quadrivalent meningococcal glycoconjugate vaccine in children 2-10 years of age. Vaccine 28: 657-663.

12. Jackson LA, Baxter R, Reisinger K, Karsten A, Shah J, et al. (2009) Phase III comparison of an investigational quadrivalent meningococcal conjugate vaccine with the licensed meningococcal ACWY conjugate vaccine in adolescents. Clin Infect Dis 49: e1-10.

13. Reisinger KS, Baxter R, Block SL, Shah J, Bedell L, et al. (2009) Quadrivalent meningococcal vaccination of adults: phase III comparison of an investigational conjugate vaccine, MenACWY-CRM, with the licensed vaccine, Menactra. Clin Vaccine Immunol 16: 1810-1815.

14. Snape MD, Perrett KP, Ford KJ, John TM, Pace D, et al. (2008) Immunogenicity of a tetravalent meningococcal glycoconjugate vaccine in infants: a randomized controlled trial. JAMA 299: 173-184.

15. Stokley S, Cohn A, Dorell C, Hariri S, Yankey D, et al. (2011) Adolescent vaccination-coverage levels in the United States: 2006-2009. Pediatrics 128: 1078-1086.

16. Gasparini R, Conversano M, Bona G, Gabutti G, Anemona A, et al. (2010) Randomized trial on the safety, tolerability, and immunogenicity of MenACWY-CRM, an investigational quadrivalent meningococcal glycoconjugate vaccine, administered concomitantly with a combined tetanus, reduced diphtheria, and acellular pertussis vaccine in adolescents and young adults. Clin Vaccine Immunol 17: 537-544.

17. Arguedas A, Soley C, Loaiza C, Rincon G, Guevara S, et al. (2010) Safety and immunogenicity of one dose of MenACWY-CRM, an investigational quadrivalent meningococcal glycoconjugate vaccine, when administered to adolescents concomitantly or sequentially with Tdap and HPV vaccines. Vaccine 28: 3171-3179.

18. Borrow R, Balmer P, Miller E (2005) Meningococcal surrogates of protection--serum bactericidal antibody activity. Vaccine 23: 2222-2227.

19. Mee RW (1984) Confidence bounds for the difference between two probabilities. Biometrics 40: 1175-1176.

20. Principi N, Esposito S (2013) Adolescents and vaccines in the western world. Vaccine 31: 5366-5374.

21. Rand CM, Shone LP, Albertin C, Auinger P, Klein JD, et al. (2007) National health care visit patterns of adolescents: implications for delivery of new adolescent vaccines. Arch Pediatr Adolesc Med 161: 252-259. 